\title{
NKG2D SIGNALING AND HOST DEFENSE AFTER MOUSE HEPATITIS VIRUS INFECTION OF THE CENTRAL NERVOUS SYSTEM
}

\author{
Kevin B. Walsh, Melissa B. Lodoen, Lewis L. Lanier, \\ and Thomas E. Lane*
}

\section{INTRODUCTION}

Natural killer (NK) cells and CD8 T cells exhibit potent anti-viral effector responses after infection with a wide variety of viruses. Through secretion of cytokines as well as cytolytic activity, these cells are capable of muting viral replication and reducing the amount of virus present within infected tissue. In recent years, additional activating and inhibitory receptors have been discovered that control both $\mathrm{NK}$ cell and $\mathrm{CD} 8^{+} \mathrm{T}$-cell activation in response to stress, transformation, and/or infection. In this study, the expression and functional role of the activating receptor NKG2D and its corresponding ligands were determined in viral infection of the central nervous system (CNS). Our data highlight a previously unappreciated role for NKG2D ligand-induced signaling in host defense after viral infection of the CNS by enhancing T-cell effector function and regulating immune-cell trafficking.

\section{THE NKG2D RECEPTOR AND ITS LIGANDS}

NKG2D is a type II transmembrane-anchored glycoprotein that is a member of the C-type lectin superfamily. ${ }^{1}$ NKG2D receptor ligation by cell-surface glycoprotein ligands that are structurally related to MHC class I molecules results in activation of lymphocytes such as natural killer (NK) cells, $\mathrm{CD}^{+}{ }^{\mathrm{T}}$ cells, and $\gamma \delta-\mathrm{TcR}^{+} \mathrm{T}$ cells. ${ }^{2,3}$ The known mouse NKG2D ligands include the retinoic acid early inducible-1 (RAE-1) proteins (RAE-1 $\alpha$, $\beta, \gamma, \delta$, and $\varepsilon)^{4}$, minor histocompatibility antigen $\mathrm{H} 60,{ }^{4}$ and murine UL16-binding protein-like transcript-1 (MULT1) glycoprotein, ${ }^{5}$ which are expressed by cells undergoing cellular stress such as viral infection or transformation.

*Kevin B. Walsh, Thomas E. Lane, University of California, Irvine, California 92697-3900. Melissa B. Lodoen, Lewis L. Lanier, University of California, San Francisco, California 94143-0414. 
A.

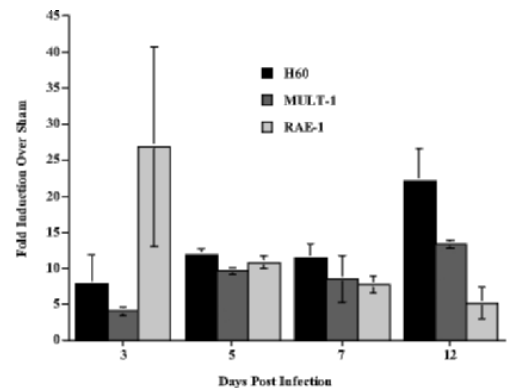

B.

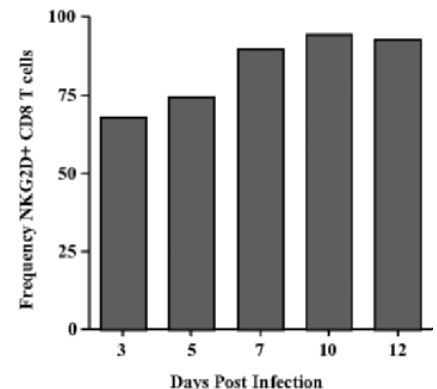

Figure 1. (A) Increased expression of NKG2D ligand mRNA transcripts within the brain after infection with MHV as determined by quantitative PCR. Ligand transcripts were not detected in the brains of sham-infected mice. (B) Flow cytometric analysis demonstrating that the majority of $\mathrm{CD}^{+} \mathrm{T}$ cells infiltrating into the brain express the NKG2D receptor after MHV infection. Data presented represent the average frequency (percentage) of CD8+ T cells present within the brain on which the NKG2D receptor was detected. Data shown represent two separate experiments with a minimum of three mice per time point.

\section{MHV INFECTION OF THE CNS}

Mouse hepatitis virus (MHV) is a positive-strand RNA virus that is a member of the Coronaviridae family. ${ }^{6}$ Instillation of MHV into the central nervous system (CNS) of susceptible mice results in an acute encephalomyelitis, followed by a chronic demyelinating disease. ${ }^{7}$ Both $\mathrm{CD}^{+}$and $\mathrm{CD}^{+} \mathrm{T}$ cells are important in reducing viral burden through secretion of anti-viral cytokines, such as interferon gamma. ${ }^{8,9}$ In addition, cytolytic activity by virus-specific $\mathrm{CD} 8^{+} \mathrm{T}$ cells is also necessary for viral clearance. ${ }^{10}$

\section{NKG2D RECEPTOR AND LIGAND EXPRESSION}

NKG2D ligand mRNA transcripts were upregulated within the brains at days $3,5,7$, and 12 after intracranial (i.c.) infection of BALB/c mice with MHV (Figure 1A). Expression of NKG2D receptor was detected on NK cells entering the CNS early after MHV infection. NKG2D receptor was also detected on $\mathrm{CD}^{+} \mathrm{T}$ cells and the frequency of $\mathrm{CD}^{+} \mathrm{T}$ cells expressing the receptor increased over time and ultimately peaked at day 10 p.i. (Figure 1B). These data indicate that NKG2D ligand mRNA and receptor are expressed during the course of MHV infection of the CNS.

\section{NKG2D NEUTRALIZATION IN IMMUNOCOMPETENT MICE}

NKG2D neutralization in MHV-infected BALB/c mice resulted in a dramatic decrease in survival compared with mice treated with a control antibody (Figure 2A). Mice treated with anti-NKG2D had increased viral titers at 7 and 12 days p.i., when compared with control mice. Moreover, anti-NKG2D treatment did result in a reduction in NK cells, T cells, and macrophages. IFN- $\gamma$ secretion was also reduced in anti-NKG2D treated mice and 
A.

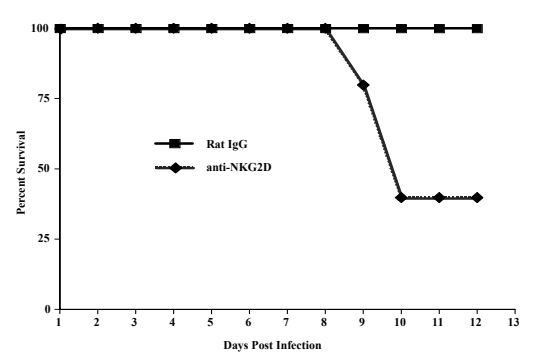

B

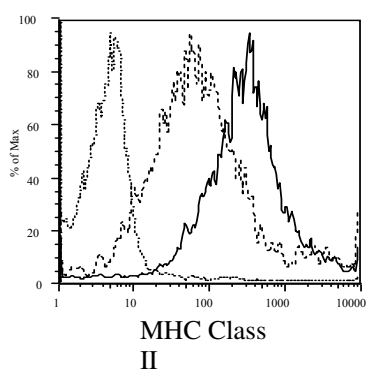

Figure 2. (A) Survival of BALB/c mice infected i.c. with MHV and treated with $100 \mu \mathrm{g}$ of rat $\operatorname{IgG}$ (control) or anti-NKG2D at 2, 5, and 10 days p.i. Data presented represent two separate experiments with a minimum of 10 mice per experimental group. (B) MHC class II expression on microglia (CD45 ${ }^{\text {low }} \mathrm{F} 480+$ ) at 12 days p.i. in MHV-infected BALB/c mice treated with rat IgG (solid line) or anti-NKG2D (dashed line). Staining from sham-infected mice is indicated by the dotted line. Shown are representative histograms.

this was reflected by a decrease in MHC class II staining of microglia (Figure 2B). These data demonstrate that NKG2D signaling is required for generation of an efficient immune response in the CNS of immunocompetent mice infected with MHV.

\section{CONCLUSIONS}

This study demonstrated that after MHV infection, NKG2D ligands are up-regulated within the CNS and infiltrating NK cells and $\mathrm{CD} 8^{+} \mathrm{T}$ cells express the NKG2D receptor. NKG2D neutralization in BALB/c mice resulted in reduced survival and diminished immune cell infiltration accompanied by decreased IFN- $\gamma$ secretion that correlated with increased viral titers in the CNS. In addition, anti-NKG2D neutralization hampered the host's ability to mount an efficient immune response, demonstrating that NKG2D signaling is important in this model of viral-induced CNS disease.

\section{ACKNOWLEDGMENTS}

This work was supported by NIH grants CA89189 and CA095137 to L.L.L. T.E.L. was supported by NIH grants NS41249 and NS18146. L.L.L. is an American Cancer Society Research Professor.

\section{REFERENCES}

1. D. H. Raulet, Roles of the NKG2D immunoreceptor and its ligands, Nat. Rev. Immunol. 3, 781-790 (2003). 
2. A. Diefenbach, A. M. Jamieson, S. D. Liu, N. Shastri, and D. H. Raulet, Ligands for the murine NKG2D receptor: expression by tumor cells and activation of NK cells and macrophages, Nat. Immunol. 1, 119-126 (2000).

3. A. M. Jamieson, A. Diefenbach, C. W. McMahon, N. Xiong, J. R. Carlyle, and D. H. Raulet, The role of the NKG2D immunoreceptor in immune cell activation and natural killing, Immunity 17, 19-29 (2002).

4. A. Cerwenka, A. B. Bakker, T. McClanahan, J. Wagner, J. Wu, J. H. Phillips, and L. L. Lanier, Retinoic acid early inducible genes define a ligand family for the activating NKG2D receptor in mice, Immunity 12, 721-727 (2000).

5. L. N. Carayannopoulos, O. V. Naidenko, D. H. Fremont, and W. M. Yokoyama, Cutting edge: murine UL16-binding protein-like transcript 1: a newly described transcript encoding a high-affinity ligand for murine NKG2D, J. Immunol. 169, 4079-4083 (2002).

6. S. R. Perlman, T. E. Lane, and M. J. Buchmeier, in: Effects of Microbes on the Immune System, edited by M. W. Cunningham and R. S. Fujinami (Lippincott Williams \& Wilkins, Philadelphia, 1999), pp. 331-348.

7. F. S. Cheever, J. B. Daniels, A. M. Pappenheimer, and O. T. Bailey, A murine virus (JHM) causing disseminated encephalomyelitis with extensive destruction of myelin, J. Exp. Med. 90, 181-194 (1949).

8. J. S. Williamson and S. A. Stohlman, Effective clearance of mouse hepatitis virus from the central nervous system requires both CD4+ and CD8+ T cells, J. Virol. 64, 4589-4592 (1990).

9. B. Parra, D. R. Hinton, N. W. Marten, C. C. Bergmann, M. T. Lin, C. S. Yang, and S. A. Stohlman, IFNgamma is required for viral clearance from central nervous system oligodendroglia, J. Immunol. 162, 16411647 (1999).

10. M. T. Lin, S. A. Stohlman, and D. R. Hinton, Mouse hepatitis virus is cleared from the central nervous systems of mice lacking perforin-mediated cytolysis, J. Virol. 71, 383-391 (1997). 